\title{
CROMOSOMAS DE MALVÁCEAS
}

\author{
AVELIANO FERNÁNDEZ ${ }^{12}$, ANTONIO KRAPOVICKAS ${ }^{1}$, GRACIELA LAVI A ${ }^{13}$ \& GUILLERMO SEIJO ${ }^{2}$
}

Summary: Fernandez, A., A. Krapovickas, G. Lavia \& G. Seijo. 2003. Chromosomes of Malvaceae. Bonplandia 12(1-4): 141-145. ISSN: 0524-0476.

Chromosome numbers are reported for 50 collections of Malvaceae from Argentina, Bolivia, Brasil, Chile, Mexico and Paraguay representing 17 genera and 40 species. First chromosome counts are reported for the following 28 species: Cienfuegosia conciliata $2 n=20$, Hibiscus striatus $2 \mathrm{n}=52, H$. adscensionis $2 \mathrm{n}=72$, Phragmocarpidium Heringeri $2 \mathrm{n}=50$, Malachra radiata $2 \mathrm{n}=56$, Pavonia Friesii $2 \mathrm{n}=56$, P. fruticosa $2 \mathrm{n}=56$, P. subrotunda $2 \mathrm{n}=56$, Monteiroa Reitzii $2 \mathrm{n}=20$, Tarasa nototrichoides $2 \mathrm{n}=10$, Tropidococcus pinnatipartitus $2 \mathrm{n}=24$, Chstaria disssecta $2 \mathrm{n}=12$, Gaya Bordasii $2 \mathrm{n}=12$, Pseudabutilon cinereum $2 \mathrm{n}=16$, Abutilon fluviatile $2 \mathrm{n}=16, A$. laxum $2 \mathrm{n}=16$, Wissadula Fadyenii $2 \mathrm{n}=14$, Sida vespertina $2 \mathrm{n}=14$, S. Glaziovii $2 \mathrm{n}=28$, S. glomerata $2 \mathrm{n}=14$, S. hirsutissima $2 \mathrm{n}=28$, S. planicaulis $2 \mathrm{n}=28$, S. Rodrigoi $2 \mathrm{n}=28$, S. ulmifolia $2 \mathrm{n}=28$, S. Hatschbachii $2 \mathrm{n}=42$, S. Reitzii $2 \mathrm{n}=42$, S. aggregata $2 \mathrm{n}=16$, S. rufescens $2 \mathrm{n}=32$.

Key words: Chromosome numbers, ploidy.

Resumen. Fernández, A., A. Krapovickas, G. Lavia \& G. Seijo. 2003. Cromosomas de Malváceas. Bonplandia 12(1-4): 141-145. ISSN: 0524-0476.

En este trabajo se presentan los números cromosómicos de 50 accesiones de Malvaceae de Argentina, Bolivia, Brasil, Chile, México y Paraguay, las mismas representan 17 géneros y 40 especies. Para las siguientes 28 especies se presenta el número cromosomico por primera vez: Cienfuegosia conciliata $2 n=20$, Hibiscus striatus $2 n=52, H$. adscensionis $2 n=72$, Phragmocarpidium Heringeri $2 \mathrm{n}=50$, Malachra radiata $2 \mathrm{n}=56$, Pavonia Friesii $2 \mathrm{n}=56, P$. fruticosa $2 \mathrm{n}=56$, P. subrotunda $2 \mathrm{n}=56$, Monteiroa Reitzii $2 \mathrm{n}=20$, Tarasa nototrichoides $2 \mathrm{n}=10$, Tropidococcus pinnatipartitus $2 \mathrm{n}=24$, Cristaria disssecta $2 \mathrm{n}=12$, Gaya Bordasii $2 \mathrm{n}=12$, Pseudabutilon cinereum $2 \mathrm{n}=16$, Abutilon fluviatile $2 \mathrm{n}=16$, A. laxum $2 \mathrm{n}=16$, Wissadula Fadyeni $2 \mathrm{n}=14$, Sida vespertina $2 \mathrm{n}=14, \mathrm{~S}$. Glaziovii2 $\mathrm{n}=28$, S. glomerata $2 \mathrm{n}=14, \mathrm{~S}$. hirsutissima $2 \mathrm{n}=28$, S. planicaulis $2 \mathrm{n}=28$, S. Rodrigoi, $2 \mathrm{n}=28$, S. ulmifolia $2 \mathrm{n}=28$, S. Hatschbachii $2 \mathrm{n}=42$, S. Reitzii $2 n=42, S$. aggregata $2 n=16, S$. rufescens $2 n=32$.

Palabras clave: Números cromosómicos, ploidía.

Los cromosomas son reconocidos por su importancia en los estudios taxonómicos y evolutivos. El número cromosómico de las entidades en estudio, teniendo en cuenta dos variables: el número básico y el nivel de ploidía, constituye un dato relevante para la resolución de problemas taxonómicos y la comprensión de las tendencias evolutivas. A pesar de dicha

'Instituto de Botánica del Nordeste (UNNE-CONICET).

${ }^{2}$ Facultad de Ciencias Exactas y Naturales y Agrimensura (UNNE).

${ }^{3}$ Facultad de Ciencias Agrarias (UNNE). 

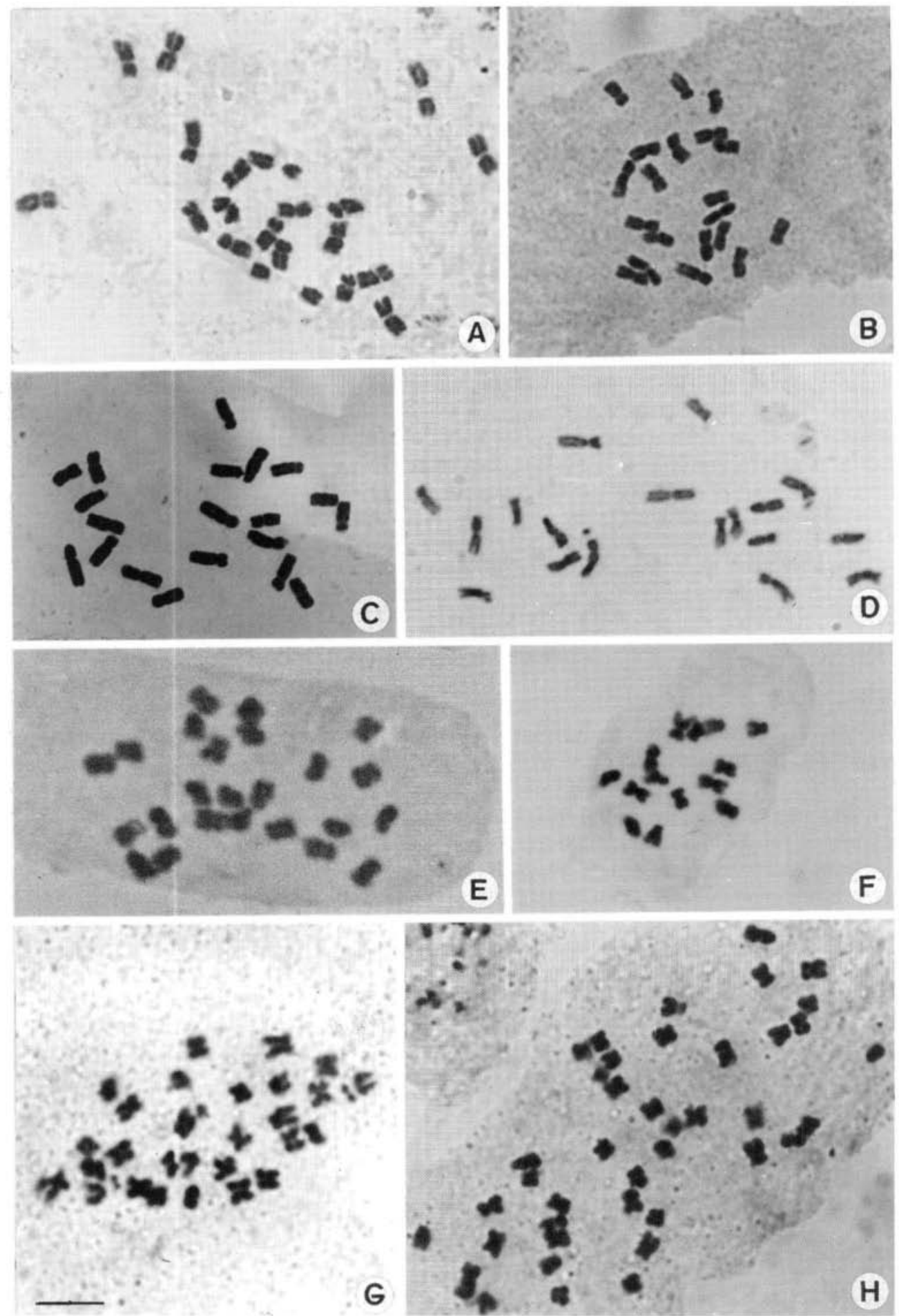

Fig. 1. A-H. Cromosomas mitóticos de Malváceas. A: Cienfuegosia conciliata 2n=20. B: C. Drumondii $2 \mathrm{n}=20$. C: Monteiroa Reitiii 2n=20. D: Modiola caroliniana 2n=18. E: Tropidococcuspinnatipartitus $2 \mathrm{n}=24$. F: Sida glomerata $2 \mathrm{n}=14$. G: S. planicaulis $2 \mathrm{n}=28$. H: S. Reitzii $2 \mathrm{n}=42$. Escala=5um. 
relevancia, sólo se conoce el número cromosómico del $25 \%$ de las angiospermas.

La familia Malvaceae, de distribución cosmopolita, incluye 130 géneros con aproximadamente 1600 especies. Aunque se han realizado muchos estudios citogenéticos en Gossypium y Malva, el conocimiento cariológico del resto de la familia es escaso. Los números cromosómicos conocidos en Malvaceae varían desde $2 \mathrm{n}=10$ en especies de distintos géneros hasta $2 \mathrm{n}=196$ en especies de Abelmoschus, existiendo diferentes números básicos: 5, 6, 7, 8, 10, 11, 12, 13, 15, 17, 18, 19, 20, 29, 36 (Fedorov 1969, Goldblatt 1981, 1984, 1985, 1988, Goldblatt and Johnson 1990, 1991, 1994, 1996, 1998, 2000, Moore 1968, 1972, 1973, 1974, 1977, Ornduff 1967); convirtiéndola en una familia de interés para estudios citogenéticos y evolutivos.

En este trabajo, se presenta el número cromosómico de 40 especies de Malváceas, con el objetivo de ampliar el conocimiento cromosómico de esta familia y contribuir a la delimitación taxonómica de algunas especies.

El material estudiado y los resultados obtenidos se muestran en la Tabla 1 y Figura 1. Los ejemplares fueron clasificados por uno de los autores (A.Krapovickas) y se encuentran depositados en el Herbario del IBONE (CTES). Con respecto a los coleccionistas, las abreviaturas utilizadas son: C: V. Chonono, G: W.C. Gregory, H: G. Hatschbach, K: A
Krapovickas, L: G. Lavia, M: Matuda, Me: X. Menhofer, Mu: T. Muñoz, P: T.M. Pedersen, S: A. Schinini, So: J.C. Solomon, T: S.G. Tressens, Tr: H. Troiani, Z: O. Zöllner. En cuanto a los países de origen del material estudiado las abreviaturas utilizadas son las siguientes: AR: Argentina, BO: Bolivia, BR: Brasil, $\mathrm{CH}$ : Chile, MX: México, PY: Paraguay.

Para realizar los recuentos cromosómicos, se utilizaron raíces obtenidas de semillas germinadas en cajas de petri, las cuales fueron pretratadas en 8 oxiquinoleína, durante 3 horas a temperatura de laboratorio, fijadas en 5 partes de etanol absoluto y una parte de ácido láctico y conservadas en etanol $70^{\circ}$. La coloración se realizó mediante la técnica de Feulgen.

Se presenta el número cromosómico de 50 accesiones que representan a las 40 especies, para 28 de ellas es el primer recuento cromosómico, y para las 12 restantes son confirmaciones de recuentos anteriores.

Los números cromosómicos obtenidos varían desde $2 \mathrm{n}=10$ para Sphaeralcea y Tarasa hasta $2 \mathrm{n}=72$ en Hibiscus, existiendo diferentes números básicos conocidos $(x=5,6,7,8,9$, $10,12,13,14)$ y uno nuevo $(\mathrm{x}=25)$, y diferentes niveles de ploidía: $2 \mathrm{x}, 4 \mathrm{x}, 6 \mathrm{x}, 8 \mathrm{x}$, lo cual estaría indicando la diversidad cromosómica existente y la posible importancia que habrían tenido los cambios cromosómicos numéricos en la diversificación de la familia.

Tabla 1. Especies estudiadas, número cromosómico, coleccionista y localidad. Asterisco indica recuento nuevo.

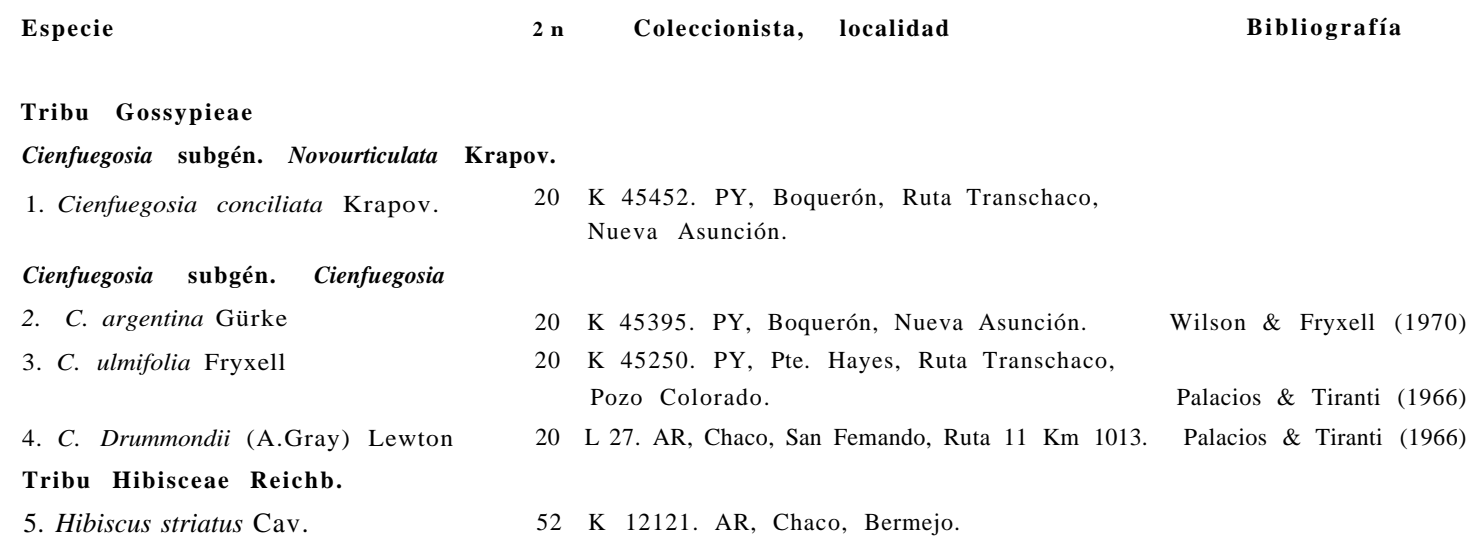




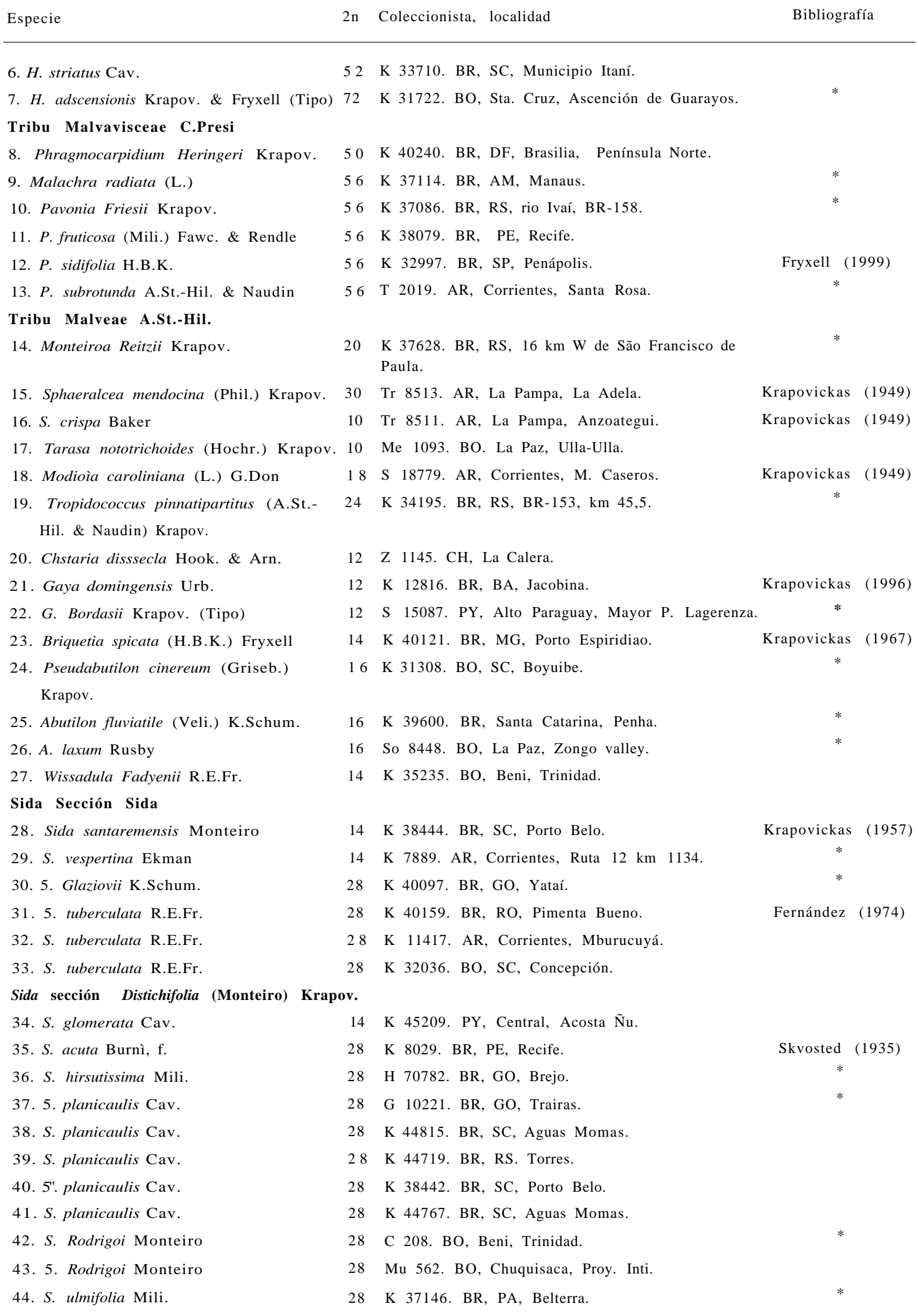


Especie

45. 5. Halschbachii Krapov.

47. S. Reitzii Krapov.

48. S. Reitzii Krapov.

Sida sección Muticae C.Presi

49. 5". aggregata C.Presi

Sida sección Nelavagae Borss.Waalk.

50. S. rufescens A.St.-Hil. 2n Coleccionista, localidad

Bibliografía

42 P 13756. BR, SC, Rio Negrinho.

42 K 44837. BR, SC, Santo Amaro da Emperatriz.

42 K 44847. BR, SC, Rio do Sui.

16 M 27870. MX, México.

\section{Bibliografía}

FEDOROV, A.A. (Ed.). 1969. Chromosome numbers of flowering plants. Komarov Botanical Institute, Academy of Sciences, USSR (Russia), Leningrad.

FERNÁNDEZ, A. 1974. Recuentos cromosómicos en Malváceas. Bol. Soc. Argent. Bot. 15: 403-410.

FRYXELL, P.A. 1999. Pavonia Cavanilles (Malvaceae). F1. Neotrop. Monogr. 76.

GOLDBLATT, P. (Ed.). 1981. Index to plant chromosome numbers 1975-1978. Monographs in Systematic Botany from the Missouri Botanical Garden 5:i-vii + 1-553.

. (Ed.). 1984. Index to plant chromosome numbers 1979-1981. Monographs in Systematic Botany from the Missouri Botanical Garden 8:i-viii + 1-427.

- (Ed.). 1985.Index to plant chromosome numbers 1982-1983. Monographs in Systematic Botany from the Missouri Botanical Garden 13:i-viii + 1-224.

. (Ed.). 1988. Index to plant chromosome numbers 1984-1985. Monographs in Systematic Botany from the Missouri Botanical Garden 23 : i-viii + 1-264.

— D.E. Johnson (Eds.). 1990. Index to plant chromosome numbers 1986-1987. Monographs in Systematic Botany from the Missouri Botanical Garden 30:i-viii + 1-243.

- (Eds.). 1991. Index to plant chromosome numbers 1988-1989. Monographs in Systematic Botany from the Missouri Botanical Garden 40:i-viii + 1-238.

- (Eds.). 1994. Index to plant chromosome numbers 1990-1991. Monographs in Systematic Botany from the Missouri Botanical Garden 51 :i-ix $+1-267$.

-. (Eds.). 1996. Index to plant chromosome numbers 1992-1993. Monographs in Systematic Botany from the Missouri Botanical Garden 58:i-x + 1-276.

—. (Eds.). 1998. Index to plant chromosome numbers 1994-1995. Monographs in Systematic Botany from the Missouri Botanical Garden 69:i- ix + 1-208.

. (Eds.). 2000. Index to plant chromosome numbers 1996-1997. Monographs in Systematic Botany from the Missouri Botanical Garden 81:1-188.

KRAPOVICKAS, A. 1949. Relación entre número cromosomico y área en el género Modiolastrum (Malvaceae). Lilloa 19: 121-125.

1957. Números cromosómicos de Malváceas americanas de la Tribu Malveae. Rev. Agron. Noroeste Arg. 2(2):245-260.

1967. Notas citotaxonómicas sobre Malveae. Kurtziana 4:29-37.

1996. Sinopsis del géneo Gaya (Malvaceae). Bonplandia 9:57-87.

MOORE, R.J. (Ed.). 1968. Index to plant chromosome numbers for 1966. Regnum Vegetabile 55:1-126.

. (Ed.). 1972. Index to plant chromosome numbers for 1970. Regnum Vegetabile 84:1-134.

. (Ed.). 1973. Index to plant chromosome numbers for 1967-1971. Regnum Vegetabile 90:1-539.

(Ed.). 1974. Index to plant chromosome numbers for 1972. Regnum Vegetabile 91:1-108.

(Ed.). 1977. Index to plant chromosome numbers for 1973-1974. Regnum Vegetabile 96:1-257.

ORNDUFF, R. (Ed.). 1967. Index to plant chromosome numbers for 1965. Regnum Vegetabile 50:1-128.

PALACIOS, R. \& I. TIRANTI. 1966. Número cromosomico de especies argentinas de Cienfuegosia Cav. (Malváceas). Boletín № 40. INTA.

WILSON, F.D. \& P.A. FRYXELL 1970. Meiotic chromosomes of Cienfuegosia species and hybrids and Hampea species (Malvaceae). Bull. Torrey Bot. Club. 97:367-376.

SKOVSTED. A. 1935. Chromosome numbers in the Malvaceae. I. J. Genetics 31:263-296.

1941. Chromosome numbers in the Malvaceae. II. C. R. Lab. Carlsb. Physiol.. 23 :195-240.

Original recibido el 1 de agosto de 2003; aceptado el 6 de octubre de 2003. 